\title{
THE EFFECT OF RELIGIOSITY ON DECISION MAKING IN SELF-DRIVING CARS: THE CASE OF "THE ETHICAL KNOB"
}

\author{
Kiana Stephen, Texas Christian University, kiana.stephen@tcu.edu \\ Beata M. Jones, Texas Christian University, b.jones@tcu.edu
}

\begin{abstract}
This study focuses on the ethical dilemmas of self-driving cars and how religiosity levels affect consumers' perceptions of morality. The research examines how religiosity affects consumers' comfort levels and moral perceptions of the "ethical knob" and the "ethical knob's" settings, which would allow the passenger of a self-driving car to determine how the car reacts in an unavoidable accident. The study does not support hypotheses concerning the effects of religiosity; however, the vast majority of respondents regardless of religiosity believed the Impartial setting was most moral, and they were most comfortable with it. This study concludes that religiosity levels alone do not affect consumers" moral perceptions of the "ethical knob" and the "ethical knob" settings, and it exposes some interesting disconnects. One would believe that more religious individuals would prefer the Impartial setting over those who are not religious due to the moral principles laid out by their religion, but the study does not support this assumption. It is likely that overall cultural differences affect consumers' moral perceptions rather than one element of culture. We must conduct more research on society's cultural elements to fully understand the moral perceptions involved and how they will affect the future of self-driving cars.
\end{abstract}

Keywords: Ethical decision-making, Self-driving cars, Ethical knob, Religiosity

\section{INTRODUCTION}

In March of 2018, a self-driving car operated by Uber hit and killed a woman in Tempe, Arizona. The woman was walking on the shoulder of the road when the self-driving car struck her at approximately 40 miles per hour (Wakabayashi, 2018). This story is a reminder that self-driving cars are not accident-proof and can still be dangerous. Many people are advocates of self-driving cars because they bring numerous benefits to society, and they will transform our lives. The most common argument is that roads will be safer for all, saving thousands of lives. According to a study by Thompson (2016), if roughly 90 percent of the automobiles on American roads were driverless, the number of accidents a year would drop from 6 million to 1.3 million. Traffic and fuel emissions also will become more efficient. If Americans adopted autonomous cars, it would reduce $\mathrm{CO}_{2}$ emissions from cars by approximately 300 million tons a year (Thompson, 2016). According to Ozimek (2014), the sum of all automobile deaths, medical and work loss, accident injuries, non-injury crashes, and time spent in the car cost the United States around \$1.23 trillion. This figure suggests that if self-driving cars reduced accidents to 1.3 million a year, the United States would save about $\$ 964$ billion annually.

Governments and consumers have high incentives to implement self-driving cars world-wide; however, society must address many issues before making self-driving cars readily available to consumers on public roads. The Federal Ministry of Transport and Digital Infrastructure in Germany recently released the "Ethics Commission: Automated and Connected Driving Report," which outlines ethical rules for connected vehicular traffic. One of the rules states, "In hazardous situations that prove to be unavoidable, despite all technological precautions being taken, the protection of human life enjoys top priority in a balancing of legally protected interests" (Federal Ministry of Transport and Digital Infrastructure, 2017, p. 11). Other countries such as the United States have begun regulating the self-driving car industry as well. On September 12th, 2018, the "National Highway and Transportation Safety Administration released $A$ Vision for Safety 2.0, the new federal guidelines for automated driving systems" "Autonomous Vehicles: Self-Driving," 2018). 


\section{Issues in Information Systems \\ Volume 21, Issue 1, pp. 74-90, 2020}

Because self-driving cars are a growing phenomenon in society, several academic researchers have focused on the ethical implications of autonomous vehicles. Specifically, many have examined the decision-making abilities of selfdriving cars in the event of unavoidable harm. Literature has proposed several solutions, including a mandatory ethics setting, and many have surveyed consumers' opinions and thoughts on these solutions. This topic is difficult and complex because while ethical dilemmas have no objective answer, our society must come to a conclusion of the "best" solution to implement in self-driving cars. In order for manufacturers to produce self-driving cars, society must arrive at an "acceptable" solution for the government and consumers. Self-driving cars could provide significant benefits to society, but if we cannot agree on the best way to handle the cars' ethics, the benefits may not materialize. This paper examines a proposed solution that each car has a customizable and changeable ethics setting, or an "ethical knob." Italian researchers Contissa, Lagioia, and Sartor (2017) proposed the ethical knob only recently, so little research currently exists on consumers' perceptions of it. The ethical knob allows for three ethical settings: Altruist, Impartial, and Egoist. Each of the settings determines whose lives the driverless car gives priority to in a situation with certain harm. This study will also include a fourth option: Random setting.

This paper examines consumers' perceptions of the ethical knob by studying the effect of religiosity on the consumers' choices. Specifically, the study measures consumers' religiosity and analyzes how moral consumers think the ethical knob settings are and which setting they prefer in relation to their religiosity levels. Religiosity is an important factor to consider when developing conclusions on user preference for ethical decision making in autonomous cars, as religion is an important driver of many people's actions. Often, religion is a construct that people will never go against; therefore, governments and manufacturers must understand if and how consumers' religion will affect driverless technology.

The remainder of the paper includes a literature review on self-driving cars, the decision- making process in selfdriving cars, the ethical dilemma of self-driving cars, the ethical knob, and religiosity's effect on decision-making and morals. After reviewing the research, the paper explains the research model used for the structure of a survey for a group of participants at a private university in Texas. The survey extrapolates information from the sample to draw conclusions about people's religiosity and its effect on their perceptions and decisions involving the ethical knob. The paper ends with a discussion of the survey data, limitations, implications drawn from the results, and conclusions.

\section{LITERATURE REVIEW}

\section{Self-Driving Cars}

The self-driving car industry is well established, worth $\$ 54.23$ billion in 2019 , and is predicted to grow to $\$ 556.67$ billion in 2026, based on current research (Garsten, 2018). Many companies, such as FedEx, Walmart, and Sysco, are already taking advantage of autonomous vehicle technology by purchasing Tesla's partial self-driving semi-truck, the Semi. PesiCo recently placed the largest Semi order, reserving 100 trucks in late 2017 (Matousek, 2018). Some studies show that people are growing more apprehensive about the technology. Nearly half of those surveyed recwently said they would never buy a fully-autonomous car, while two years prior, only 30 percent expressed this opinion (Edelstein, 2018). Based on the data, consumers are not ready to trust self-driving cars; however, many manufacturers are racing to begin producing them. Officials in California have given approval to fifty-two companies (Apple, Honda, BMW, Nissan, etc.) to test driverless vehicles on the road (Miller, 2018). Manufacturers must find a way to help consumers feel comfortable and in control while riding in autonomous cars.

Self-driving vehicles make have five different "levels" of automation, according to The US National Highway Traffic Safety Administration. These levels include cars with no automated control systems, cars with certain autonomous features such as automatic braking, and fully automated cars (National Highway Traffic Safety Administration, 2013). In this paper, self-driving cars are considered fully autonomous, replacing a human driver.

\section{Decision Making Process in Self-Driving Cars}

Self-driving cars rely on different information inputs than human drivers do. These inputs include computer vision, parallel computing, machine learning, and more. Based on the inputs, self-driving cars make calculations based on an abstraction of the real world. Of course, to have the abstraction match reality as closely as possible, more data is helpful; however, too much data may require more filtering to find the useful information, or the computer may pass 


\section{Issues in Information Systems \\ Volume 21, Issue 1, pp. 74-90, 2020}

by key points (Holstein et al., 2018). Software engineers, therefore, have the responsibility to decide which data the cars should use in the decision-making process.

Programmers take two common approaches to automate moral decision-making in artificial intelligence. They use game theory to represent decision scenarios where multiple parties have different interests. Different sequences of actions lead to certain parties gaining or losing from the scenario; thus necessitating the extention of game theory concepts in order to encompass moral behavior (Borg, Contizer, Deng, Kramer, \& Sinnott-Armstrong, 2017). Machine learning is another approach. With this approach, a programmer must "assemble a training set of moral decision problem instances labeled with human judgments of the morally correct decision(s), and allow the AI system to generalize" (Borg et al., 2017, p. 4834).

\section{Ethical Dilemmas of Self-Driving Cars}

Although self-driving cars are safer for society than human operated vehicles, automomous vehicles cannot avoid all accidents. At times, self-driving cars will have to make a moral choice even though they do not have a moral compass by nature. A common example of this situation is represented through three situations in Bonnefon, Shariff, and Rahwan (2015). A self-driving car is driving on the road when it meets pedestrians/a pedestrian in the middle of the street. The situations/options are as follows:

a) The car can stay on course and kill several pedestrians or swerve and kill one passerby

b) The car can stay on course and kill one pedestrian or swerve and kill its passenger

c) The car can stay on course and kill several pedestrians or swerve and kill its passenger.

When avoiding harm is not possible, the harm must be distributed. In the situations above, the car must have built-in moral algorithms in order to make a decision, and the moral algorithms must align with society's moral attitudes. Moral attitudes are not universal. In fact, human societies embrace many different moral doctrines, complicating how self-driving cars need to be programmed. One of the most common and accepted moral doctrines is utilitarianism or the consequentialist approach. This approach examines the outcome of the options and chooses the one with the best outcome (Shou \& Song, 2017). In situations a) and b) above, the utilitarian perspective would be in favor of killing one person (the passerby/passenger) versus many people. Another competing view is the deontological approach. The deontological approach focuses on whether "an act is consistent with a moral principle or duty" (Shou \& Song, 2017, p. 481). An example of a moral principle would be to never kill another person. From the deontological perspective, regardless of how many people could be saved, it would be morally wrong to kill another. In the situations presented above and in the ethical dilemma of self-driving cars overall, the deontological approach is difficult to apply. Selfdriving cars make decisions on how to react in an accident long before the crash occurs. Currently, humans make decisions in split seconds during accident scenarios, and they likely do not have the time to consider moral consequences.

Awad et al. (2018) dove deeper into the moral dilemmas faced by autonomous vehicles with their "Moral Machine." Their study captures the high dimensionality of the self-driving car ethical dilemma. The Moral Machine is a 'game' that allows for the worldwide collection of data about individuals' ethical preferences on autonomous vehicles in different areas of the world. The people playing the "game" choose one of two options in accident scenarios. The article focused on nine factors: saving humans versus pets, staying on course versus swerving, sparing more or fewer lives, sparing men or women, sparing young or old, sparing pedestrians who cross legally versus jaywalkers, sparing fit versus not, and sparing higher social status individuals versus lower (Awad et al., 2018).

Awad et al. (2018) gathered a large data set and developed three different clusters (Western, Eastern, and Southern) based on the location of the respondents. Based on the cluster to which the researcher assigned them, respondents allocated largely different weights to their ethical preferences, which likely resulted from differences in culture and the economies of the countries in the clusters. For example, the study found strong preference overall to spare young lives versus old lives, and it found that respondents who identified with high levels of religiosity preferred saving humans over animals.

Although the literature did not attempt to pinpoint the reason behind the findings across cultures, it provided valuable insight into the correlations for future research. Some literature alludes that individuals that identify with higher levels 


\section{Issues in Information Systems \\ Volume 21, Issue 1, pp. 74-90, 2020}

of religiosity also are more collectivistic. One study found that non-religious people have lower levels of collectivism than religious individuals (Protestants and Catholics) (Stark, 2009).

In order to resolve the disparity in moral opinions, one study suggests a mandatory ethics setting for self-driving cars (Gogoll \& Muller, 2017). Based on literature, people tend to believe that the best option is to program all self-driving cars with utilitarian ethics; however, the same people would prefer to ride in cars programmed to protect the passengers and would not buy a car programmed otherwise (Bonnefon et al., 2016). Noothigattu et al. (2017) offer three suggestions on how to deal with the self-driving car ethical dilemma: impose a mandatory ethics setting on self-driving cars, allow manufacturers/programmers to decide, or create a personal ethics setting for passengers ("the ethical knob"), which will be the focus of the following section. Some literature suggests aggregating people's opinions on ethical dilemmas and creating a concrete approach for ethical decision making based on it (Noothigattu et al., 2017). Each of these suggestions comes with pros and cons, and manufacturers and regulators will need to manage three incompatible goals: being consistent, not causing public outrage, and not discouraging buyers (Bonnefon et al., 2016). Consumers must feel comfortable with autonomous cars' ethical decision making because a study in Korea found that as consumers' ethical expectations for autonomous vehicles increase, consumers' positive thinking about autonomous vehicles also increases. The study defined ethical expectations by observing whether consumers thought the selfdriving cars would make desirable decisions, ethical decisions, and whether the consumers trusted the ethics of autonomous cars (Ha \& Ro, 2017).

\section{The Ethical Knob}

The trolley problem is a common ethical thought experiment used for years to discuss the concept of "killing versus letting die." Ethicists established the original trolley problem as follows:

Edward is the driver of a trolley, whose brakes have just failed. On the track ahead of him are five people; the banks are so steep that they will not be able to get off the track in time. The track has a spur leading off to the right, and Edward can turn the trolley onto it. Unfortunately there is one person on the right-hand track. Edward can turn the trolley, killing the one; or he can refrain from turning the trolley, killing the five. (Thomson, 1976, p. 206)

Researchers have altered the trolley problem for many different ethical thought experiments including for self-driving cars. The problem poses the difficult idea that deciding to kill one will save multiple, but if you do not act, then you are letting five individuals die. A version of the trolley problem used for self-driving cars was stated above from Bonnefon et al. (2015).

Researchers have proposed the concept of an "ethical knob" in an attempt to solve the self- driving car ethical dilemma, and it grew from the trolley problem's ethical options. Many people fear that mandatory ethics setting in all selfdriving cars will discourage consumers from buying them since, based on studies, people prefer to ride in a vehicle that protects the passengers (Bonnefon et al., 2016). Millar (2015) discusses the problem with removing the users of self-driving cars from the decision-making process. In the event of an unavoidable accident, the situation has a direct implication on the users, and if governments and manufacturers do not consider their input, automating self-driving cars' ethical decision making threatens the moral autonomy of the consumer. To allow artificial intelligence to choose the outcomes of moral dilemmas amounts to a form of paternalism which is ethically undesirable because it excludes the user from the decision-making process (Millar, 2016).

Contissa et al. (2017) were the first to propose the concept of the "Ethical Knob" in their article. Their scenario assumes that the passenger will have the task of picking the ethical approach that the car takes in the event of an accident, and the self-driving car will only implement the ethical choice rather than make the decision. To execute this idea, the self-driving cars would be equipped with a knob with three settings (see Figure 1):

1. Altruistic mode: Gives the preference for third parties (always sacrifices the self- driving car's passenger(s) lives)

2. Impartial mode: Gives equal importance to passengers and third parties (by basing the decision of who lives on the utilitarian principle - saving the most people) 


\section{Issues in Information Systems \\ Volume 21, Issue 1, pp. 74-90, 2020}

3. Egoistic mode: Gives preference for passengers (always sacrifices third parties and pedestrians)

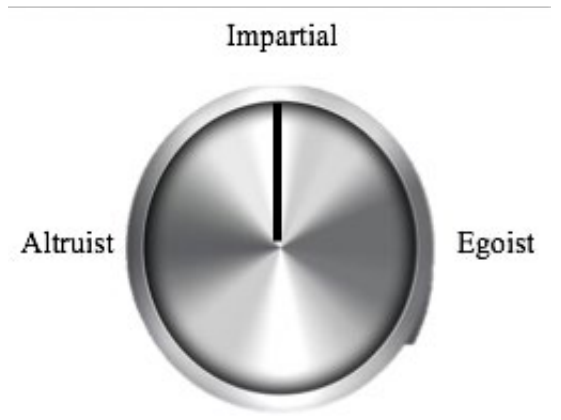

Figure 1. The Ethical Knob

If a passenger sets the knob to the Impartial mode and there is equilibrium when evaluating the situation using the utilitarian principle, the car could make the decision based on presumption or random choice. Contissa et al. (2017) discuss the possibility of the ethical knob having continuous settings to assign weights to certain people's lives. For the case of this study, the ethical knob will only have three distinct options, and the author has added a fourth "Random" option. The random option will instruct the vehicle to pick an option randomly.

Some may question what the difference is between having cars with an ethical knob and allowing consumers to buy cars with their ethical preference pre-programmed. With the ethical knob, passengers can establish kin altruism. Setting the knob to Egoist would allow for kin altruism to take place because the self-driving car would act to protect the passenger and loved ones riding in the car as well (Contissa et al., 2017). The car's ethical setting can be changed according to the situation or the driver (Beall, 2017).

As discussed earlier, the ethical decision must be in someone's hands: the manufacturer, government, or consumer. Personal ethics setting such as the ethical knob is desirable because if it is what consumers want, manufacturers must produce self-driving cars with ethical knobs, and governments should only pass laws and regulations that align with the morals of their citizens. Ethical preferences are too diverse to create a regulation that pleases most, if not all, citizens (Gogoll \& Muller, 2017).

\section{Religiosity's Effect on Decision Making and Morals}

Literature suggests that more religious individuals generally are more likely to make deontological judgments over utilitarian/consequentialist judgements. As discussed previously, deontological ethics focuses on duty rather than the outcome. Some actions are right or wrong regardless of the consequences or results. This conviction is likely due to the belief that morality is founded on divine authority and "moral rules" found in most religions instead of reasoning (Piazza \& Landy, 2013). Some of these value frameworks include Islam's Sharia, Catholicism's Canon Law, and Judaism's Halacha (Esptein, 2010). Different rules govern different religions, but most religions share common principles. Most important to this study is the prohibition of killing and the inaction principle. The inaction principle states that consequences caused by doing something (action) are worse than the consequences caused by inaction (Zamir, 2014). In contrast, the indirectness principle prohibits an action if it uses a person as a means to an end. (Cushman, Hauser, \& Young, 2006).

Some religions have different ideas of what it means to be moral. Protestants view thoughts just as important as actions; therefore, if a person thinks of doing something immoral, then he or she is being immoral. By contrast, Judaism cares more about the actual actions (Cohen, Wu, \& Miller, 2016). This distinction becomes important when one studies the ethical knob since the thought is an important factor in the choice.

This study combines the findings of previous literature with new proposed solutions such as the ethical knob. It examines the effect of religiosity on participants' perceptions of the ethical knob settings and which they would select. Previous research provided valuable insight into the research methods and conclusions drawn from this study. 


\title{
Issues in Information Systems
}

Volume 21, Issue 1, pp. 74-90, 2020

\section{RESEARCH METHODS}

\begin{abstract}
Research Hypotheses
The researcher hypotheses test the relationship between religiosity and the settings chosen on the ethical knob as well as the perceived morality of the settings in self-driving cars.
\end{abstract}

H1: Higher levels of religiosity will lead consumers to believe the morality of the ethical knob in general is low.

As discussed by Zamir (2014), the inaction principle prohibits any action regardless of the potential outcome, and many religions incorporate this principle into their belief systems. People with high levels of religiosity will not want to choose any setting because they are taking action that may decide who lives and who dies. Also, some religions, such as Protestants, believe that the thought can be immoral without the action (Cohen et al., 2016). The ethical knob inherently causes the user to think about what to choose, and individuals with high levels of religiosity would not believe this thought to be moral.

\section{H2: Higher levels of religiosity will lead consumers to believe the Impartial setting on the ethical knob is most} moral.

As discussed previously, since individuals with higher levels of religiosity believe that the action is immoral, they will not want to decide on who to sacrifice in the case of an accident (Zamir, 2014). The Impartial setting comes closest to inaction because the car will make the decision at the moment based on the situation; therefore, the user does not take the final "action" deciding who to spare.

H3: Higher levels of religiosity will lead consumers to believe the morality of choosing the Altruist setting on the ethical knob is higher than the morality of choosing the Egoist setting.

Many religions have definitive moral principles that one must abide by, and those with higher religiosity are more likely to believe in the deontological principle (Piazza \& Landy, 2013). One of the most common laws is that one must never kill. Those with high levels of religiosity likely would find setting the ethical knob to Altruist as more moral than Egoist because Altruist would avoid harm to others, i.e., not choosing to sacrifice someone's life in a crash. The Egoist setting would be most similar to killing someone, as the passenger is sacrificing another person to save his or her life.

H4: Higher levels of religiosity will make consumers least comfortable with self-driving cars with ethical knobs set to Egoist on the road than the other three settings.

Again, many religions prohibit killing. Those with high levels of religiosity will be more comfortable if no one on the road chooses to sacrifice others using the Egoist setting. Participants who fall higher on the religiosity scale will not be comfortable on the roads if others are going against the participants' morals.

\section{H5: Higher levels of religiosity will make consumers most comfortable with self-driving cars with ethical knobs set to Impartial on the road over the other settings.}

Those with high levels of religiosity are more likely to believe that taking any action is morally wrong, especially when it involves the lives and well-being of others. The Impartial setting allows the car to choose what action to take based on the scenario rather than the passenger, so individuals who have higher levels of religiosity will favor this option on their roads because it means that others are not completing an immoral action or decision. With the Impartial setting, no one is choosing the final action to take in regard to human life.

H6: Higher levels of religiosity will make consumers less willing to buy self-driving cars with an ethical knob.

Individuals with high levels of religiosity are more likely to believe that the action is in and of itself immoral (Zamir, 2014). The action of choosing a setting on the ethical knob would be immoral because the passenger is taking an 
action that involves someone's life. These individuals will not want to buy a car that forces them to take action that makes them feel immoral.

\section{H7: Lower levels of religiosity will make consumers more likely to believe that the Impartial setting is more moral than the Random setting.}

Individuals with low levels of religiosity tend to believe in the utilitarian principle (Barak-Corren \& Bazerman, 2017). They believe it is most moral to save the most lives. The Random setting will choose any of the three options without taking the situation, number of people, etc. into account; therefore, it will not be the most utilitarian option.

\section{Methodology}

This survey study used an online questionnaire administered to students and faculty at a private Texas university as well as friends and peers of the researchers. The survey assessed the effect of religiosity on respondents' decisions regarding the "ethical knob" and its settings in self-driving cars. The survey was distributed via URL included in emails to participants. To ensure that each respondent only completes the survey once, the survey software tracked IP addresses to block duplicates. Qualtrics was used to conduct the survey, and the survey included 16 questions requiring less than 10 minutes to complete.

The Qualtrics survey gathered the data regarding participants' religiosity and its correlation to ethical decision making regarding self-driving cars. Then statistical software was used to measure the relationship between personal religiosity and its effect on the participant's preference for the ethical knob setting: Altruistic, Impartial, Egoistic, or Random. The dependent variable in this study was the setting participants believe is the most moral on the ethical knob, and the independent variable was the participants' religiosity levels. In order to measure religiosity, the survey employed an existing religiosity framework used by Barak-Corren and Bazerman (2017). To test what people would choose on the ethical knob, the researchers used slightly modified scenarios and questions from Bonnefon et al. (2016).

The participants of the survey came from varying backgrounds, as university students come from different areas of the world, genders, ages, and religions. Since religion can be a sensitive topic that some people feel uncomfortable answering questions about, the survey included other demographic questions first, and the religion questions were included at the end of the survey. Before officially launching the survey and collecting data, the researchers pilot tested the instrument. The data was collected over two weeks in early 2019.

\section{RESULTS}

Researchers cleaned the data collected from the survey by removing any unfinished survey responses, responses indicating a lack of attention, and test responses. Removing these responses resulted in a sample size of 284 respondents. The following section summarizes the analysis and findings from the survey.

\section{Demographic Data}

This survey included several questions related to demographics. To identify general information about respondents, there were questions regarding respondents' gender, age, education level, and ethnicity/ethnic origin (one question each). The survey also contained seven questions related to the level of religiosity. Using these seven questions, researchers calculated one, the final measure of religiosity per respondent from one to seven. The final measure was calculated by averaging the responses from the seven questions. Researchers used this final measure, referred to as "full religiosity measure," several times throughout the analysis. The measure can range from one to seven - one being the lowest level of religiosity and seven being the highest level of religiosity. With a Cronbach's Alpha of .947, the seven-question scale had high reliability and internal consistency. Table 1 illustrates the demographic data as percentages out of the 284 respondents. 


\section{Issues in Information Systems}

Volume 21, Issue 1, pp. 74-90, 2020

Table 1. Demographic Data

\begin{tabular}{|c|c|c|c|c|c|c|c|c|c|}
\hline \multirow{2}{*}{ D12 } & \multirow{2}{*}{ Gender } & Female & Male & & & & & & \\
\hline & & $44.7 \%$ & $55.3 \%$ & & & & & & \\
\hline \multirow{2}{*}{ D13 } & \multirow{2}{*}{ Age } & $18-22$ & $22-30$ & $31-40$ & $41-50$ & $51-60$ & Over 60 & & \\
\hline & & $75.7 \%$ & $3.2 \%$ & $1.1 \%$ & $2.8 \%$ & $7.7 \%$ & $9.5 \%$ & & \\
\hline \multirow{2}{*}{ D14 } & \multirow{2}{*}{$\begin{array}{c}\text { Education } \\
\text { Level }\end{array}$} & High-school & 1 year college & 2 years college & 3 years college & 4 years college & Bachelor & \begin{tabular}{|l|} 
Master \\
\end{tabular} & Doctoral \\
\hline & & $9.5 \%$ & $26.8 \%$ & $34.9 \%$ & $6.0 \%$ & $2.8 \%$ & $5.3 \%$ & $7.4 \%$ & $7.4 \%$ \\
\hline \multirow{2}{*}{ D16 } & \multirow{2}{*}{$\begin{array}{c}\text { Ethnicity/ } \\
\text { Ethnic } \\
\text { Origin }\end{array}$} & White & Hispanic/Latino & Black/AA & Native American & Asian/PI & Other & & \\
\hline & & $84.5 \%$ & $8.1 \%$ & $1.4 \%$ & $0.0 \%$ & $4.9 \%$ & $1.1 \%$ & & \\
\hline \multirow[b]{2}{*}{ R5-11 } & \multirow{2}{*}{$\begin{array}{c}\text { Full } \\
\text { Religiosity } \\
\text { Measure }\end{array}$} & Low 1-2.99 & Neutral 3-4.99 & High 5-7 & & & & & \\
\hline & & $15.8 \%$ & $27.8 \%$ & $56.3 \%$ & & & & & \\
\hline
\end{tabular}

"D\# - Demographic variables' associated question number in the survey

"R\# - Religiosity variable's associated question numbers in the survey

By looking at the data in Table 1, one can note that the majority of respondents are 18-30 years of age and have completed two years of college or less (roughly $78.9 \%$ and $71.2 \%$, respectively). Additionally, the vast majority of survey respondents are white (roughly $84.5 \%$ ) with little representation of other ethnicities. Lastly, those with low levels of religiosity were under represented (roughly $15.8 \%$ of respondents) while those with high levels of religiosity contributed to over half the responses (roughly 56.3\%). This over-representation of high levels of religiosity can likely be contributed to the fact the university where the study was completed identifies as a faith-based institution.

In order to add to the discussion of the perceived morality of self-driving cars and the ethical knob, the researchers analyzed the correlations between the demographic variables and perceived morality of the ethical knob in general. In order to detect any differences in perceived morality of the ethical knob (Q1) between the two genders (D12), the researchers conducted a Two Sample (assuming unequal variances) $t$-Test assuming a 95\% confidence interval. The test found a significant difference in means between male and female respondents ( $\mathrm{p}$-value $=0.035$ ). Males on average rated the morality of the ethical knob higher than females did (means $=49.44$ and 43.24 respectively).

Since the majority of respondents were 18-22 years of age, the comparison between means for the other age groups was difficult; therefore, the researchers split the sample into two groups: Generation Z/Millennials (18-40) and Generation X through the Silent Generation (41-60+). Researchers conducted a Two Sample (assuming unequal variances) $t$-Test to analyze the difference between the mean ratings of the perceived morality of the ethical knob of these two groups. The results indicate no significant difference in means between the two age groups ( $\mathrm{p}$-value $=0.425$ ). Lastly, the researchers analyzed the education level to observe if there was an effect on the perceived morality of the ethical knob in general. Again, since the individual education level groups have different sample sizes, the sample was split into those that have had two years or less of college and those that have more than two years of college, and researchers conducted a Two Sample (assuming unequal variances) t-Test. There was no significant difference between the mean ratings of the perceived morality of the ethical knob in general ( $p$-value $=0.15$ ). Overall, the demographic variables collected did not seem to have an effect on the perceived morality of the ethical knob other than the gender variable. Some evidence suggests that males perceive the ethical knob as more moral than females do.

\section{H1: Higher levels of religiosity will lead consumers to believe the morality of the ethical knob in general is low.}

In order to address and analyze the first hypothesis, researchers analyzed the seven questions related to religiosity (R5-R11), and one question about the perceived morality of the ethical knob in general was asked (Q1). The researchers used a simple linear correlation to analyze whether there is a relationship between level of religiosity and the perceived morality of the ethical knob. First, a scatter plot was created using the full religiosity measure for each respondent as the independent variable and the perceived morality of the ethical knob as the dependent variable (see Figure 2). The findings indicate no linear relationships between the two variables, as the data points are scattered. To confirm this conclusion, a trend line was added to the scatter plot using linear regression, and it was evident there was no relationship, as the correlation coefficient was almost zero $\left(\mathrm{R}^{2}=-.0004\right)$. Based on this analysis, Hypothesis 1 was 


\section{Issues in Information Systems}

Volume 21, Issue 1, pp. 74-90, 2020

not confirmed. There is no evidence that higher levels of religiosity lead consumers to believe the morality of the ethical knob in general is low.

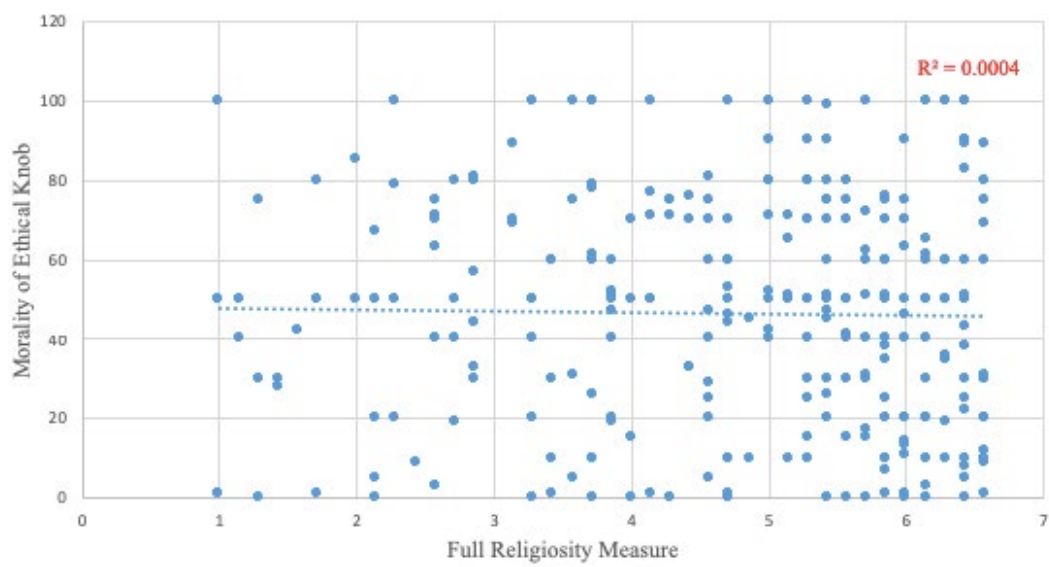

Figure 2. Correlation Between Religiosity Level and Perceived Morality of the Ethical Knob

\section{H2: Higher levels of religiosity will lead consumers to believe the Impartial setting on the ethical knob is most moral.}

To test the validity of Hypothesis 2, the researchers used one question (with four sub-questions) regarding the morality of each setting on the ethical knob (Q2), and seven questions regarding the religiosity of the respondent were analyzed to create one full religiosity measure per respondent (R5-R11). First, the sample was broken into two groups: those that rated the Impartial setting (II) higher than the other three settings (most moral) and those that did not. From these groups, the full religiosity measures were extrapolated for each respondent and averaged to get the mean religiosity level for each of the two groups. These means were compared using a Two Sample (assuming unequal variances) tTest with a $95 \%$ confidence interval. Table 2 summarizes the output of this analysis.

Table 2. H2 Output

\begin{tabular}{|c|c|c|}
\hline \multicolumn{3}{|c|}{ t-Test: Two-Sample Assuming Unequal Variances } \\
\hline & $\begin{array}{c}\text { Impartial } \\
\text { Rated Highest }\end{array}$ & $\begin{array}{c}\text { Impartial } \\
\text { NOT Rated } \\
\text { Highest }\end{array}$ \\
\hline Mean Religiosity Level & 4.7903 & 4.6883 \\
\hline Variance & 1.9956 & 3.1648 \\
\hline Observations & 217 & 67 \\
\hline Hypothesized Mean Difference & 0 & \\
\hline df & 91 & \\
\hline t Stat & 0.4267 & \\
\hline $\mathrm{P}(\mathrm{T}<=\mathrm{t})$ One-Tail & 0.3353 & \\
\hline t Critical One-Tail & 1.6618 & \\
\hline $\mathrm{P}(\mathrm{T}<=\mathrm{t})$ Two-Tail & 0.6706 & \\
\hline t Critical Two-Tail & 1.9864 & \\
\hline
\end{tabular}

As Table 2 indicates, the means of the religiosity levels of the two groups were similar. The t-Test confirmed that the slight difference in means is not significant and is due to random chance, as the p-value was greater than 0.05 ( $\mathrm{p}$-value $=0.34$ ). By observing the differences between the average religiosity level in the two groups, one can gain insight into whether religiosity level affects respondents' views on the morality of the ethical knob settings. For example, if the 


\section{Issues in Information Systems}

Volume 21, Issue 1, pp. 74-90, 2020

means were significantly different, one could conclude that religiosity levels may have an impact on the respondents' moral perceptions. Since there was no significant difference in the two groups' means, one can conclude that higher levels of religiosity do not lead consumers to believe that the Impartial setting is most moral. Hypothesis 2 was not confirmed.

\section{H3: Higher levels of religiosity will lead consumers to believe the morality of choosing the Altruist setting on the ethical knob is higher than the morality of choosing the Egoist setting.}

In order to analyze Hypothesis 3, the researcher analyzed the seven questions related to religiosity (R5-R11) to calculate a full religiosity measure for each participant. Once a full religiosity measure was calculated for each participant, the sample was split into three groups based on religiosity level. The three groups were those with low levels of religiosity (1-2.99 full religiosity measure), those with neutral levels of religiosity (3-4.99 full religiosity measure), and those with high levels of religiosity (5-7 full religiosity measure). One question (with four subquestions) related to the perceived morality of the four settings was also used (Q2). Specifically, the sub-questions related to the Egoist setting (Q2, setting I) and the Altruist setting (Q2, setting III) were analyzed. The points given to these two settings (1-100, 100 being perceived as most moral) were identified for each person in the three groups. Then, for each group, the mean ratings were calculated for each of the two settings. These means were compared used a Paired Two-Sample t-Test for each group. Finally, the corresponding P-values were analyzed (see Table 3).

Table 3. H3 Output

\begin{tabular}{|l|r|r|r|r|}
\hline & Mean Egoist & Mean Altruist & $\begin{array}{c}\text { Difference in Means } \\
\text { (Absolute Value) }\end{array}$ & \multicolumn{1}{c|}{ p-value } \\
\hline Low Religiosity & 14.40 & 12.24 & 2.16 & 0.305 \\
\hline Neutral & 10.234 & 14.16 & 3.92 & 0.029 \\
\hline High Religiosity & 10.91 & 18.95 & 8.04 & $2.35 \mathrm{E}-05$ \\
\hline
\end{tabular}

Table 3 reveals differences in the perceived morality/rating of the Egoist setting and the Altruist setting in all three groups. The Paired Two-Sample t-Tests confirms that the differences in means are significant for the Neutral and High Religiosity groups ( $\mathrm{p}$-value $=0.029$ and $\mathrm{p}$-value $=2.35 \mathrm{E}-05$, respectively); however, for the Low Religiosity group, the difference in means is not significant ( $\mathrm{p}$-value $=0.305$ ). A column chart was created to visualize the mean differences. The religiosity groups are on the $\mathrm{x}$-axis, and the average perceived morality ratings of the two settings are on the y-axis (see Figure 3).

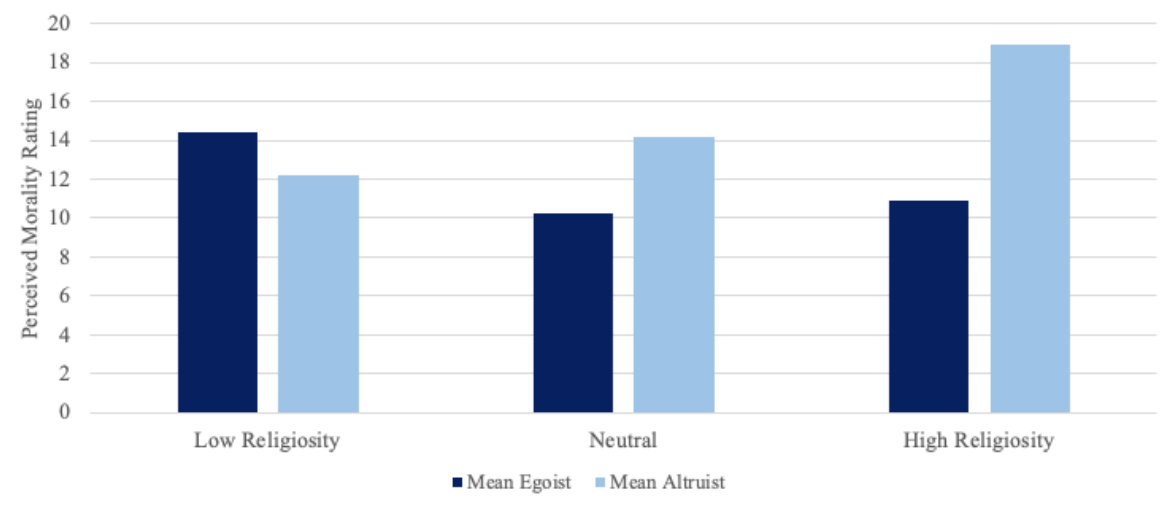

Figure 3. Egoist and Altruist Setting Rating Means for Three Different Groups Based on Religiosity Level

Figure 3 reveals differences in the morality ratings for the Egoist and Altruist settings; however, the size of the difference varies according to the religiosity group. For the low religiosity group's means, the mean of the Egoist setting was higher than the mean of the Altruist setting. For the Neutral and High Religiosity Groups, the means for the perceived morality of the Altruist setting was higher than the means for the Egoist setting. Also, the difference between the means was greater for the High Religiosity group than the Neutral group (8.04 and 3.92, respectively). 


\section{Issues in Information Systems \\ Volume 21, Issue 1, pp. 74-90, 2020}

This information may confirm Hypothesis 3. Since the difference between the means grows as the level of religiosity grows, the evidence may support the idea that higher levels of religiosity will lead consumers to believe the morality of choosing the Altruist setting on the ethical knob is higher than the morality of choosing the Egoist setting. However, the difference in means for the Low Religiosity group was not statistically significant, so it is necessary to conduct more research.

\section{H4: Higher levels of religiosity will make consumers least comfortable with self-driving cars with ethical knobs set to Egoist on the road than the other three settings.}

To analyze Hypothesis 4, the researchers used seven questions on religiosity (R5-R11) to calculate one, full religiosity measure for each respondent, and one question (with four sub-questions, Q3) regarding the participants' comfortability of each ethical knob setting. Similar to the H2 analysis, the sample was broken into two groups: those least comfortable with the ethical knob set to Egoist (setting I) and those least comfortable with one of the other settings. For each group, the full religiosity measures for each respondent were extrapolated and averaged to get the mean religiosity level for the group. To analyze the difference in the means of the two groups, the researchers used a Two-Sample (assuming unequal variances) t-Test with a $95 \%$ confidence interval. Table 4 presents the output of the test.

Table 4. H4 Output

\begin{tabular}{|c|c|c|}
\hline \multicolumn{3}{|c|}{ t-Test: Two-Sample Assuming Unequal Variances } \\
\hline & $\begin{array}{c}\text { Least } \\
\text { Comfortable } \\
\text { with Egoist } \\
\text { Setting }\end{array}$ & $\begin{array}{c}\text { Least } \\
\text { Comfortable } \\
\text { with Another } \\
\text { Setting }\end{array}$ \\
\hline Mean Religiosity Level & 4.8866 & 4.7474 \\
\hline Variance & 2.0368 & 2.2859 \\
\hline Observations & 34 & 250 \\
\hline Hypothesized Mean Difference & 0 & \\
\hline df & 44 & \\
\hline t Stat & 0.5294 & \\
\hline $\mathrm{P}(\mathrm{T}<=\mathrm{t})$ One-Tail & 0.2996 & \\
\hline t Critical One-Tail & 1.6802 & \\
\hline $\mathrm{P}(\mathrm{T}<=\mathrm{t})$ Two-Tail & 0.5992 & \\
\hline t Critical Two-Tail & 2.0154 & \\
\hline
\end{tabular}

Table 4 shows that the religiosity level means for the two groups were similar. The slight difference in means was not significant ( $p$-value $=0.29$ ). Analyzing whether there is a significant difference in the means of the two groups would help confirm or reject Hypothesis 4 because a significant difference would indicate that religiosity levels may have affected the responses of the participants. There was no significant difference in the groups' mean religiosity level; therefore, one cannot conclude that higher levels of religiosity will make consumers least comfortable with self-driving cars with ethical knobs set to Egoist on the road than the other three settings. The findings did not confirm Hypothesis 4.

\section{H5: Higher levels of religiosity will make consumers most comfortable with self-driving cars with ethical knobs set to Impartial on the road over the other settings.}

To analyze Hypothesis 5, the researchers used seven questions related to religiosity (R5-R11) as well as one question (with four sub-questions) related to respondents' comfort with the ethical knob settings (Q3). From the seven religiosity questions, one measure of religiosity was calculated for each participant ("full religiosity measure"). First, the sample was separated into two independent groups: individuals most comfortable with the Impartial setting (setting II) and individuals most comfortable with one of the other three settings. Then the researchers extrapolated each individual's full religiosity measure, and the researchers calculated the average religiosity measure for both groups. With this information, a Two-Sample (assuming unequal variances) t-Test using a 95\% confidence interval was conducted. Table 5 summarizes the results of this test. 


\section{Issues in Information Systems}

Volume 21, Issue 1, pp. 74-90, 2020

Table 5. H5 Output

\begin{tabular}{|c|c|c|}
\hline \multicolumn{3}{|c|}{ t-Test: Two-Sample Assuming Unequal Variances } \\
\hline & \begin{tabular}{|c|} 
Most \\
Comfortable \\
with Impartial \\
Setting
\end{tabular} & $\begin{array}{c}\text { Most } \\
\text { Comfortable } \\
\text { with Another } \\
\text { Setting }\end{array}$ \\
\hline Mean Religiosity Level & 4.7349 & 4.8551 \\
\hline Variance & 2.1513 & 2.5861 \\
\hline Observations & 215 & 69 \\
\hline Hypothesized Mean Difference & 0 & \\
\hline df & 107 & \\
\hline t Stat & -0.5515 & \\
\hline$P(T<=t)$ One-Tail & 0.2912 & \\
\hline t Critical One-Tail & 1.6592 & \\
\hline $\mathrm{P}(\mathrm{T}<=\mathrm{t})$ Two-Tail & 0.5824 & \\
\hline t Critical Two-Tail & 1.9824 & \\
\hline
\end{tabular}

Similar to the results of Hypotheses 2 and 4, the religiosity level means were similar across the two groups. The TwoSample t-Test indicates that the slight difference in means was not significant ( $p$-value $=0.29)$. Significant differences in the mean religiosity levels would likely indicate that the level of religiosity plays a role in the respondents' level of comfort with the ethical knob settings. Since there was not a significant difference between the means, Hypothesis 5 could not be confirmed. Higher levels of religiosity did not make consumers most comfortable with self-driving cars with ethical knobs set to Impartial on the road over the other settings.

\section{H6: Higher levels of religiosity will make consumers less willing to buy self-driving cars with an ethical knob.}

In order to test Hypothesis 6, the researchers used seven questions relating to religiosity levels (R5-R11) along with one question regarding respondents' willingness to buy a self-driving car with an ethical knob installed (Q4). From the seven religiosity questions, one measure of religiosity from one to seven was calculated for each survey respondent ("full religiosity measure"). First, a scatter plot was created using the full religiosity measure as the independent variable and the level of willingness to buy a self-driving car with an ethical knob (1-100) as the dependent variable. The scatterplot helps viewers visualize the relationship between the two variables; however, the results indicate there was no relationship because a pattern is not apparent in Figure 4. To confirm this observation, a linear regression was completed. The correlation coefficient was almost zero $\left(\mathrm{R}^{2}=3 \mathrm{e}-5\right)$, so there was no relationship. Because there is no correlation between religiosity levels and willingness to buy a self-driving car with an ethical knob, Hypothesis 6 was not confirmed.

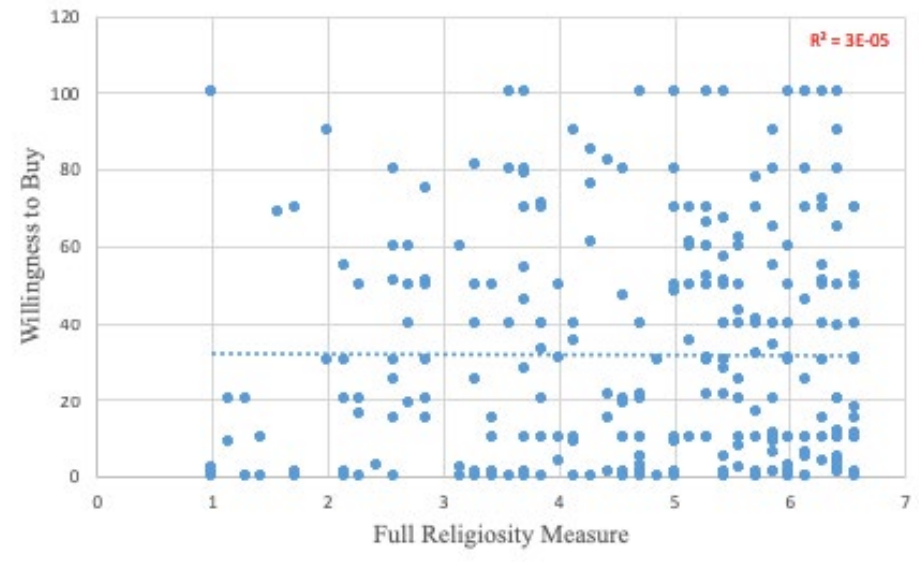

Figure 4. Correlation Between Religiosity Level and Willigness to Buy a Self-driving Car with an Ethical Knob 


\section{Issues in Information Systems}

Volume 21, Issue 1, pp. 74-90, 2020

\section{H7: Lower levels of religiosity will make consumers more likely to believe that the Impartial setting is more moral than the Random setting.}

To analyze Hypothesis 7, the researchers used seven religiosity questions (R5-R11) to calculate one religiosity measure for each individual (1-7). After calculating these measures, the sample was split into three independent groups: those with low levels of religiosity (1-2.99), those with neutral levels of religiosity (3-4.99), and those with high levels of religiosity (5-7). For all groups, each individual's ratings from 1-100 were analyzed for the Impartial setting (Q2, setting II) and the Random setting (Q2, setting IV). The mean ratings for the Impartial setting and the Random setting were calculated for all three groups separately. Then, the researchers compared these means using Paired Two-Sample t-Tests for each group, and the corresponding p-values were analyzed (see Table 6).

Table 6. H7 Output

\begin{tabular}{|l|r|r|r|}
\hline & Mean Impartial & Mean Random & p-value \\
\hline Low Religiosity & 59.56 & 13.80 & $8.03 \mathrm{E}-10$ \\
\hline Neutral & 64 & 11.61 & $1.08 \mathrm{E}-24$ \\
\hline High Religiosity & 57.58 & 12.56 & $7.25 \mathrm{E}-38$ \\
\hline
\end{tabular}

As Table 6 indicates, the researchers found no significant differences in the perceived morality of the Impartial setting means and the perceived morality of the Random setting means across all three groups. The Paired Two-Sample tTests confirmed that the differences in the means for the settings were all significant. For the Low Religiosity group, the difference in means was significant ( $\mathrm{p}$-value $=8.03 \mathrm{e}-10)$. This finding is the same for the Neutral group ( $\mathrm{p}$-value $=1.08 \mathrm{e}-24)$ and the High Religiosity group ( $\mathrm{p}$-value $=7.25 \mathrm{e}-38$ ). To visualize the mean differences, the researchers created a column chart with the religiosity groups on the $\mathrm{x}$-axis and the average perceived morality ratings on the $\mathrm{y}$ axis (see Figure 5).

As Figure 5 illustrates, there are large differences between the average perceived morality ratings for the Impartial setting and the Random setting for all three groups; however, it also illustrates that religiosity levels do not cause these differences. Since all three groups show a much lower perceived morality rating for the Random setting, Hypothesis 7 was not confirmed. Lower levels of religiosity do not affect consumers' beliefs that the Impartial setting is more moral than the Random setting.

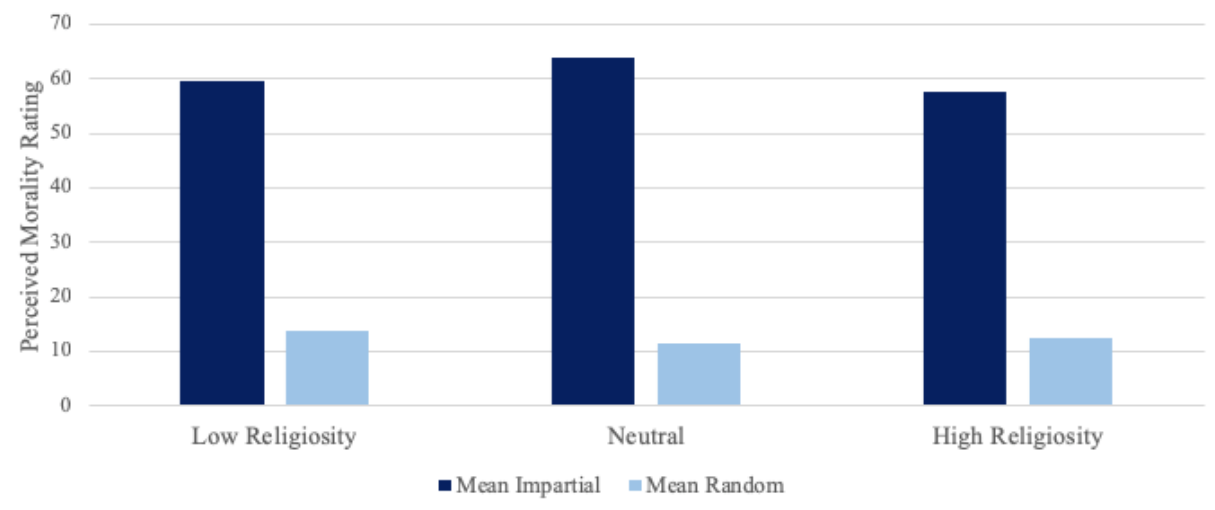

Figure 5. Impartial and Random Setting Rating Means for Three Different Groups Based On Religiosity Levels

\section{DISCUSSION}

Out of the seven hypotheses presented, six were not confirmed (H1-2 \& H4-7), and one may be supported with further research (H3); however, there were some other interesting findings from the survey. An analysis of the survey results made it apparent that while religiosity levels may not have affected individuals' answers, there were trends across a majority of individuals. First, for Hypothesis 2, it was evident that the majority of respondents (regardless of religiosity 


\section{Issues in Information Systems \\ Volume 21, Issue 1, pp. 74-90, 2020}

level) believed that the Impartial setting was most moral. Roughly $76.6 \%$ of respondents identified the Impartial setting as the most moral out of the four settings. Along with this finding for Hypothesis 3, the mean ratings for the two settings (Altruist and Egoist) were low for all three religiosity groups. Every mean was below 20 (out of 100), suggesting that respondents perceived the morality of the Egoist and Altruist setting as relatively low. This finding emphasizes that the majority of individuals believe the Impartial setting is most moral. Along with the perceived morality of the Impartial setting, a majority of individuals were also most comfortable with the Impartial setting. About $75.7 \%$ of respondents rated the Impartial setting the highest when asked how comfortable they were with each setting. Lastly, when asked about their willingness to buy a self-driving car with an ethical knob, roughly $74.6 \%$ of respondents rated their willingness to buy as less than 50 out of 100 . The majority of people would not be comfortable buying an autonomous car with an ethical knob.

Although religiosity levels did not seem to affect the perceived morality and comfort of the four ethical knob settings, other factors may have influenced participants' choices. In this study, evidence shows males may perceive the morality of the ethical knob in general as higher than females do. Although there may be a correlation based on gender, it does not imply causation, so more research should be conducted regarding this relationship. The study did not see any relationship between the other demographic variables such as age and education level, but a broader force may be at work - underlying moral principles. These moral principles are not universal, and it is important for future studies to identify what affects individuals' morality. A survey published by Nature concluded that many moral principles that guide people's behavior vary by country of residence (Maxmen, 2018).

The Moral Machine Experiment studied the many moral decisions that self-driving cars must make (young vs. old, animals vs. humans, etc.) over 40 million people and across 233 countries. After thorough analysis, the researchers discovered that the majority of individuals agreed only on sparing humans over pets and groups of people over individuals. Other than that, they shared no universal moral code. So, the researchers divided the responses into three groups that answered relatively similarly on the moral conundrums. The three groups were as follows: North American and European nations; Japan, Indonesia, and Pakistan; and Central and South American nations along with France. The biggest conclusion for this study is that so many cultural factors comprise our moral perceptions. For example, the level of economic inequality in individuals' culture may affect their decision to save a homeless person, and the different religions present in other countries also may have had an effect on their decisions (Awad et al., 2018). This finding is interesting because in our study, the researchers looked at the effect of the overall religiosity levels of respondents regardless of the specific religion people follow. In the future, the researcher may find it useful to study the different religions' affect as well as the many other aspects that shape cultures, such as power distance, forms of government, economic systems, history, and daily life.

\section{LIMITATIONS}

There are several limitations of this study that are worthy of note. First, the sample used in the survey analysis had little diversity. The majority of respondents were white and had an education level of fewer than three years of college ( $84.5 \%$ and $71.2 \%$ respectively). Also, the majority of respondents were $18-22$ years of age (75.7\%). Although these demographic variables did not appear to have an effect on the results of the study, it is important to have broad representation from all demographic groups to take into account all variables that could affect the results and, therefore, ensure more accurate conclusions. Another limitation of this study would be the personal pressure respondents may feel to respond a certain way when nswering questions about morals. Some participants likely have a perception of what the "right" answer is even if their first instinct would be to choose a different answer. When respondents feel like answering a question a certain way may define their character, they are less likely to choose the answer they really believe over the "right" answer. Also, respondents' understanding of self-driving cars, what decisions they must make, etc. is an important factor in the analysis of their decisions that researchers did not take into account in this study. Those who more thoroughly understand self-driving cars and the underlying algorithms and decision making involved, may have different answers than those who do not understand anything about self-driving cars. Those who understand the many nuances and underlying dilemmas autonomous cars are tied to may believe the morality of the ethical knob is higher. This concept is another area of research that would prove important because if education about self-driving cars would make consumers understand the ethical implications, it may be worth investing in for the future. 


\section{Issues in Information Systems \\ Volume 21, Issue 1, pp. 74-90, 2020}

Lastly, the trolley problem and the ethical implications of self-driving cars may not belong together. Some ethicists argue that the trolley problem is an oversimplified way to think about a complicated problem. For example, one article stated that the trolley problems are "unrealistic and unrepresentative of the moral situations people encounter in the real world" and "they do not elicit the same psychological processes as other moral situations" (Bauman, McGraw, Bartels, \& Warren, 2014, p. 536). Some people believe that rather than focusing on the concept of the intentionality of self-driving cars, we should spend that time focusing on teaching the cars to avoid harm in the first place (Baram, 2019). For future studies, it may be helpful to use other ethical problems that take more complexities into account to more closely resemble the real world.

\section{IMPLICATIONS}

This research findings have implications for individual consumers, manufacturers of self-driving cars, and governments around the world. First, it is important for consumers to understand their moral preferences and how their morality relates to the ethical conundrums of self-driving cars. Although many believe that God or a higher being is responsible for what happens in their lives, this belief may be challenged by the adoption of self-driving cars because someone must program the decisions a self-driving car makes. This study taught us that religiosity may not play a role in consumers' ethical decisions in relation to the ethical knob because there was not an option for consumers to not choose anything on the knob, which would be the equivalent to surrendering the outcome to God or a higher being. In many of the proposed solutions to the ethical dilemmas posed by autonomous cars, there is not an option that would be similar to how accidents are dealt with now. Often, the driver makes the decision in milliseconds, but with selfdriving cars, someone with little understanding of the specific situation must make the decision far in advance in order to program the car. In the future, consumers will need to think about their preferences and how the introduction of self-driving cars will affect them.

Until all cultures embrace a universal law of how to program cars to deal with unavoidable accidents, manufacturers need to understand user preferences, and what they are willing to buy. This study shows that the level of religiosity may not affect consumer preferences when it comes to the ethical knob, but it may be worth understanding consumers' religions when it comes to other proposed solutions. Overall, manufacturers cannot begin selling self-driving cars until there is a large enough population that is willing to buy what they are selling. This realization means that consumers must believe how the car makes decisions is morally right. Manufacturers should focus on providing detailed information to consumers on how their cars make decisions and what that means for ethical dilemmas. They should also focus on how cars get information from their surroundings to make the best decisions.

Lastly, this study has implications for governments around the world. Eventually, governments must regulate selfdriving cars and car manufacturers. This study showed that levels of religiosity may not have an effect on consumers' ethical choices; however, other studies suggest that specific religions and cultures may have different moral perceptions. This finding may perhaps suggest that governments of each country should be in charge of selecting the best moral solution for their citizens. In order to do so, governments should invest in researching their population and their preferences. From this research, governments can more thoroughly understand what factors affect the citizens' moral perceptions and how they should take those perceptions into account when identifying a universal solution for self-driving cars.

\section{CONCLUSIONS}

Self-driving cars pose significant opportunities to societies if adopted successfully; however, there are currently too many ethical dilemmas in the mix to fully implement the technology. This research has proven that humans' morality is not one-size-fits-all, and we are far from understanding what shapes our individual perceptions. Specifically, the level of religiosity does not influence humans' moral perceptions regarding self-driving cars and ethical-knob.

Although religiosity alone does not shape individuals' moral perceptions, other studies have shown that culture, as a whole, does. For future studies, it is important to explore culture more thoroughly. With consistent research, it may become apparent that there are a few aspects that drastically affect our views; however, we may never fully understand our cultures and how they shape our moral values because cultures are complex concepts. In this case, it is important for governments and manufacturers to create solutions that individuals will be comfortable with. At this point, there 


\section{Issues in Information Systems \\ Volume 21, Issue 1, pp. 74-90, 2020}

are too many moral dilemmas to create one solution, but if we want to realize the benefits of self-driving cars, something must transpire to move forward with this new technology.

\section{REFERENCES}

"Autonomous vehicles: Self-driving vehicles enacted legislation." (2018). National Conference of State Legislatures. Retrieved from http://www.ncsl.org/research/transportation/autonomous-vehicles-self-driving-vehiclesenacted-legislation.aspx

Awad E., Bonnefon, J., Dsouza, S., Henrich, J., Kim, R., Rahwan, I., Schulz, J., \& Shariff, A. (2018). The moral machine experiment. Nature, 563(7729), 59-64.

Barak-Corren, N., \& Bazerman, M. (2017). Is saving lives your task or God's? Religiosity, belief in god, and moral judgment. Judgment and Decision Making, 12(3), 280-296.

Baram, M. (2019). Why the trolley dilemma is a terrible model for trying to make self-driving cars safer. Fast Company. Retrieved from https://www.fastcompany.com/90308968/why- the-trolley-dilemma-is-aterrible-model-for-trying-to-make-self-driving-cars-safer

Bauman, C., McGraw, P., Bartels, D., \& Warren, C. (2014). Revisiting external validity: Concerns about trolley problems and other sacrificial dilemmas in moral psychology. Social and Personality Psychology Compass, $8(9), 536-554$.

Beall, A. (2017). The car you can program to sacrifice you in a crash. New Scientist. 11.

Bonnefon, J., Rahwan, I., \& Shariff, A. (2015). Autonomous vehicles need experimental ethics: are we ready for utilitarian cars?. ArXiv:1510.03346v1, 1-15.

Bonnefon, J., Rahwan, I., \& Shariff, A. (2016). The social dilemma of autonomous vehicles. Science, 352(6293), 1573-1576.

Borg, J., Conitzer, V., Deng, Y., Kramer, M., \& Sinnott-Armstrong, W. (2017). Moral decision making frameworks for artificial intelligence. Association for the Advancement of Artificial Intelligence Proceedings 31, 4831-4835.

Cohen, A., Wu, M., \& Miller, J. (2016). Religion and culture: Individualism and collectivism in the East and West. Journal of Cross-Cultural Psychology, 47(9), 1236-1249.

Contissa, G., Lagioia, F., \& Sartor, G. (2017). The ethical knob: ethically-customisable automated vehicles and the law. Artificial Intelligence and Law, 25(3), 365-378.

Cushman, F., Hauser, M., \& Young, L. (2006). The role of conscious reasoning and intuition in moral judgment testing three principles of harm. Psychological Science, 17(12), 1082-1089.

Edelstein, S. (2018). Consumers have become more apprehensive about self-driving cars, study says. The Drive. Retrieved from http://www.thedrive.com/tech/22939/consumers- have-become-more-apprehensiveabout-self-driving-cars-study-says

Esptein, G. (2010). Good without God: What a billion nonreligious people do believe. New York: HarperCollins.

Federal Ministry of Transport and Digital Infrastructure. (2017). Ethics commission: Automated and connected driving. Berlin: BMVI.

Garsten, E. (2018). Sharp growth in autonomous car market value predicted but may be stalled by rise in consumer fear. Forbes. Retrieved from https://www.forbes.com/sites/edgarsten/2018/08/13/sharp-growth-inautonomous-car-market-value-predicted-but-may-be-stalled-by-rise-in-consumer-fear/\#ca1947617c2c

Gogoll, J., \& Muller, J. (2017). Autonomous cars: In favor of a mandatory ethics setting. Science and Engineering Ethics, 23(3), 81-700.

Ha, Y., \& Ro, Y. (2017). A factor analysis of consumer expectations for autonomous cars. Journal of Computer Information Systems, 59(1), 52-60.

Holstein, T., Dodig-Crnkovic, G., \& Pelliccione, P. (2018). Ethical and social aspects of self-driving cars. ArXiv: 1802.04103v1, 1-11.

Matousek, M. (2018). Tesla has a new customer for its electric Semi - here are all the companies that have ordered the big rig. Business Insider. Retrieved from https://www.businessinsider.com/companies-thatordered-tesla-semi-2017-12

Maxmen, A. (2018). Self-driving car dilemmas reveal that moral choices are not universal. Nature, 562(7728), 469470.

Millar, J. (2015). Technology as moral proxy: Autonomy and paternalism by design. IEEE International Symposium on Ethics in Science, Technology, and Engineering (47-55). Chicago, IL: IEEE 


\section{Issues in Information Systems}

Volume 21, Issue 1, pp. 74-90, 2020

Millar, J. (2016). An ethics evaluation tool for automating ethical decision-making in robots and self-driving cars. Applied Artificial Intelligence, 30(8), 787-809.

Miller, A. (2018). Some of the companies that are working on driverless car technology. abcNews. Retrieved from https://abcnews.go.com/US/companies-working-driverless-car-technology/story?id=53872985

National Highway Traffic Safety Administration. (2013). Preliminary statement of policy concerning automated vehicles.Retrieved from http://www.nhtsa.gov/staticfiles/rulemaking/pdf/Automated_Vehicles_Policy. Pdf

Noothigattu, R., Awad, E., Dsouza, S., Gaikwad, N., Procaccia, A., Rahwan, I., \& Ravikumar, P. (2017). A votingbased system for ethical decision making. Association for the Advancement of Artificial Intelligence Proceedings, 32, 1-13.

Ozimek, A. (2014). The massive economic benefits of self-driving cars. Forbes. Retrieved from https://www.forbes.com/sites/modeledbehavior/2014/11/08/the-massive-economic-benefits-of-self-drivingcars/\#35fb34233273

Piazza, J. \& Landy, F. (2013). "Lean not on your own understanding": Belief that morality is founded on divine authority and non-utilitarian moral judgments. Judgment and Decision Making, 8(6), 639-661.

Shou, Y. \& Song, F. (2017). Decisions in moral dilemmas: The influence of subjective beliefs in outcome probabilities. Judgment and Decision Making, 12(5), 481-490.

Stark, R. T. Jr. (2009). Does God matter? religion in individualistic and collectivistic personalities. School of Professional Psychology: Theses, Dissertations and Capstone Projects. 1-38.

Thompson, C. (2016). The 3-biggests ways self-driving cars will improve our lives. Business Insider. Retrieved from https://www.businessinsider.com/advantages-of-driverless-cars-2016-6

Thomson, J. (1976). Killing, letting die, and the trolley problem. The Monist: Philosophical Problems of Death, 59(2), 204-217.

Wakabayashi, D. (2018). Self-driving Uber car kills pedestrian in Arizona, where robots roam. New York Times. Retrieved from https://www.nytimes.com/2018/03/19/technology/uber-driverless-fatality.html

Zamir, E. (2014). Law, psychology, and morality: The role of loss aversion. London: Oxford University Press. 